\title{
Semirigid Ureteroscopy: Step by Step
}

\author{
Guido Giusti, MD, Silvia Proietti, MD, Moises Elias Rodríguez-Socarrás, MD, \\ Giuseppe Saitta, MD, Piera Bellinzoni, MD, and Franco Gaboardi, MD
}

\begin{abstract}
This article aims to demonstrate a step-by-step technique of semirigid ureteroscopy (URS) for the treatment of ureteral stones, urothelial tumors, and ureteral stenosis. Operating room setup, camera settings, access to the bladder, and negotiation of the ureteral orifice, lasertripsy, basketing of the stone fragments, endoscopic treatment of ureteral tumors and ureteral stenosis, flexible URS at the end of semirigid URS, and Double-J stent placement are described step by step.
\end{abstract}

Keywords: ureteroscopy, endourology, lithiasis

$\mathbf{H}$ ISTORICALLY, URETEROSCOPIC SURGERY has improved from a mere diagnostic procedure with several limits to an accurate, complex, and technologically advanced surgical procedure able to treat different stones, tumors, and ureteral strictures.

\section{Indications}

Apart from general problems related, for example, to general anesthesia or untreated urinary tract infections, ureteroscopy (URS) may be performed in all patients without any specification contraindications. ${ }^{1,2}$

The main indication for the use of the semirigid ureteroscope is the treatment of distal ureteral stones below the level of iliac vessels. For more proximal stones, although semirigid ureteroscopes could be used, the utilization of flexible ureteroscopes is nowadays encouraged because of the not negligible risk of retropulsion into the kidney. ${ }^{3}$

Moreover, semirigid URS could be performed before flexible URS. One advantage of doing so is that the whole ureter is passively dilated under direct vision while exactly realizing the compliance of the ureter to better identify the ideal ureteral access sheath (UAS) size according to the patient's anatomy. Second, especially in the case of secondlook procedures, missed fragments that migrated down into the ureter can be removed before UAS placement. As a matter of fact, friction of the UAS against fragments can produce ureteral lesions that are sometimes severe.

\section{Preoperative Preparation, Patient Positioning, and Operating Room Setting}

For all patients undergoing URS, a routine preoperative work-up should be performed, including history, physical examination, urinalysis, urine culture, complete blood count, serum creatinine evaluation, coagulation status, and plain abdominal computer CT scan or ultrasonography.

The radiological images should be displayed in the operating room (OR) and available at any time in case of doubts during the procedure.

In case of positive urine culture, antibiotic intake should start 5 days before the procedure; otherwise, the procedure should be postponed.

Patients taking antiplatelet or anticoagulant treatment should not be considered as a contraindication for URS, since this procedure demonstrates no major complications for these kind of patients. ${ }^{4}$

Informed consent should be obtained from all patients before the surgery. The patient must be apprised of the nature, risks (fever, infection, hematuria, urinary system perforation, ureteral avulsion, renal hematoma, need for antegrade ureteral access, need for surgical revision repair, stent discomfort, need for staged procedure, delayed urethral and ureteral stenosis, and failure of gaining the ureteral access) and possible alternatives of the medical procedure.

A preoperative checklist should be filled in and the side of the stone should be marked on patient's skin.

The patient is placed in lithotomy position, ensuring that all pressure points are cushioned to prevent tissue and nerve damage. ${ }^{5,6}$

Although spinal is a viable option, general anesthesia is suggested for URS for two reasons. First, because of larger tidal volume, breathing movement can be disturbing for the surgeon during navigation and lithotripsy; moreover, mechanical ventilation can be temporarily stopped when needed. ${ }^{7,8}$

Second, in the case of larger stones, the timeframe of a spinal anesthesia can be exceeded, requiring a second general anesthesia to finish the procedure.

Department of Urology, San Raffaele Hospital, Ville Turro Division, Milan, Italy. 


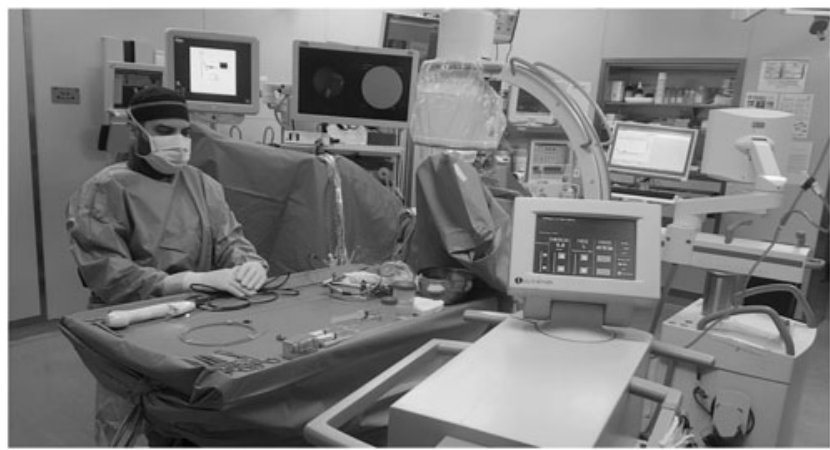

FIG. 1. Operating room setup for ureteroscopy.

All patients should receive one single dose of intravenous antibiotic prophylaxis, 1 hour before starting the surgery.

The OR setting is important. C-arm fluoroscopy should always be present and usually positioned on the left of the patient, whereas the endoscopic tower is usually on the right.

The laser machine should be placed close to the surgeon in order not to pull the laser fiber inside the working channel of the instrument inadvertently. Appropriate safety guidelines of its use must be adhered to for the safety of both the patient and OR staff.

A table should always be placed close to the patient to have all ureteroscopes and disposable equipment available, straight and easy to pick up (not hanging) thereby avoiding these instruments from inadvertently sliding out (Fig. 1).

Warm irrigation should be available in the OR and an active irrigation system should be used when an overpressure is needed for better observation of the endoscopic field.

Any additional equipment that may be required during the procedure should be prepared and be readily available before starting the case.

\section{Surgical Steps}

\section{Access to the bladder and negotiation of the ureteral orifice}

Usually, the procedure starts with cystoscopy with the double intent of placing a safety guidewire into the upper urinary tract and of excluding potential malignancy of the lower urinary tract.

Alternatively, the procedure may start directly with semirigid URS. In this case, placement of a $12 \mathrm{~F}$ Foley catheter is advocated: it should be kept indwelling and open throughout the entire procedure to guarantee a low-pressure system and to facilitate the ureteroscope's advancement.

Insertion of the semirigid ureteroscope in the urethra should always be gentle avoiding excessive bending stress on its shaft as may happen in the case of an enlarged prostate gland (Fig. 2).

Once the ureteral orifice is identified, the placement of a safety guidewire, although not compulsory, is highly advised. Which is the ideal guidewire is still under debate, with Polytetrafluoroethylene (PTFE) wires more stable but kinkable, whereas hydrophilic ones have a tendency to slip out but not kinkable and are more likely to glide past an obstruction. Our preference is for the latter keeping in mind their drawbacks.

In certain circumstances, difficulty in advancing the guidewire into the ureter may be experienced because of

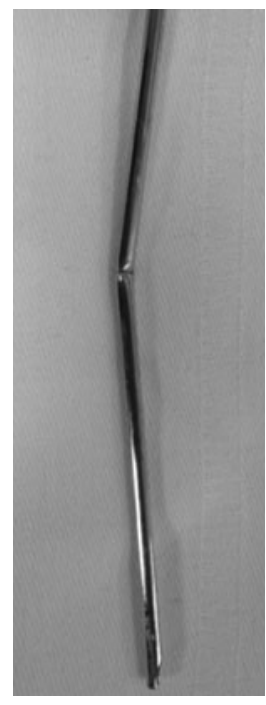

FIG. 2. Cracked semirigid ureteroscope as a result of excessive force delivered to overcome elevated and rigid bladder neck.

anatomical barriers ( $\mathrm{J}$-hooking of the distal ureter, an impacted stone, tight stricture, etc.). Angled catheters and/or guidewires can be useful in such situations.

Once the safety guidewire is secured to the drape (Fig. 3), the semirigid ureteroscope is inserted again into the bladder. According to the "railway technique," at this point a second guidewire is passed up into the collecting system to safely lead the advancement of the ureteroscope into the ureter. Alternatively, a $10 \mathrm{~F}$ dual lumen catheter may be utilized with this aim.

With newer generation slim and ultraslim semirigid ureteroscopes, negotiation of the papilla can be attempted with the instrument straight and in line with the ureter. With older and bigger ones, because of their tapered extremity, a $180^{\circ}$ rotation may be needed to overcome the curved beak at its tip. ${ }^{9}$

In the era of urogram-CT scan it is questionable whether or not a retrograde pyelography should be done systematically as advocated in the past. To limit the radiation exposure for both patients and surgeons, our suggestion, according to ALARA principle, is to perform it only when the endoscopic scenario is not clear and not in accordance with, or in the absence of, a urogram-CT scan.

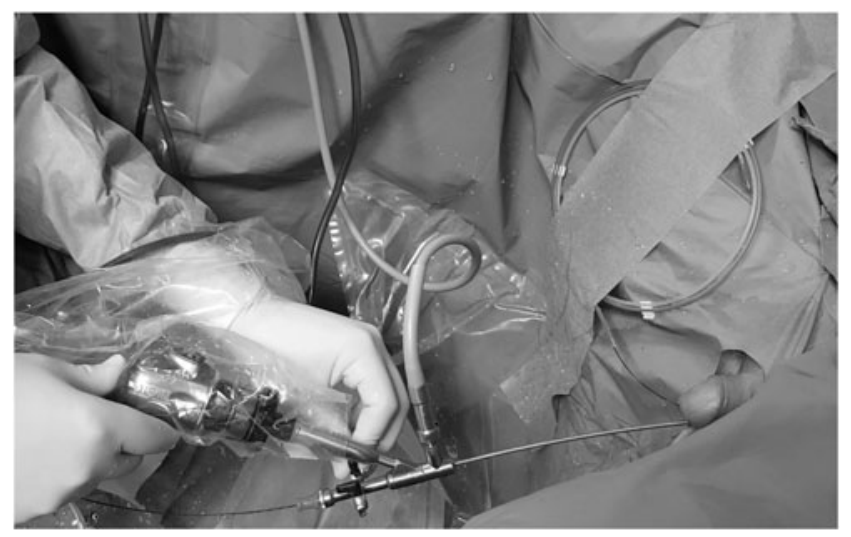

FIG. 3. Safety guidewire secured to the drape during the semirigid ureteroscope. 


\section{URS: navigation of the ureter, lithotripsy, and stenting}

When insertion of the ureteroscope is difficult because of an uncompliant anatomy, some authors suggest various techniques such as balloon and Teflon coaxial dilators. ${ }^{10}$ In our opinion, the modern endourologist should change his or her mentality: adaptation of the endourological armamentarium to a patient's anatomy should always be the strategy and not vice versa. As such, when negotiation is difficult, downsizing of the ureteroscope should be the first choice: with the newer ultraslim semirigid ureteroscope impossible negotiation is rare. Having said this, whenever excessive resistance is felt while advancing the ureteroscope, the surgeon should stop and consider temporary stenting and rescheduling the patient after 2 weeks rather than balloon dilating an uncompliant ureter.

Once into the ureter, the ureteroscope is advanced over the working wire up to the stone with irrigation gently modulated by the assistant to find a good balance between a good visibility without retropulsion. A manual handheld device is appropriate because the visibility is usually impaired by the stone's pulverization during lasertripsy. Different devices are actually available all possessing exclusive advantages and disadvantages: caution is always to be taken in manipulating these devices because clear vision cannot be obtained at the expenses of high intrarenal pressure. ${ }^{11}$

Traditionally the prevention of stones' retropulsion was carried out by entrapping the calculus into a basket before starting lithotripsy. In the past decade, special antiretropulsion devices have been developed such as the Stone Cone ${ }^{\mathrm{TM}}$ by Boston Scientific, the N-TRAP ${ }^{\mathrm{TM}}$ by Cook medical, and the Xen-X by Rocamed.

These instruments are placed and opened above the calculus to prevent retropulsion of stone fragments and facilitate fragment extraction on removal of the device. Retropulsion can reduce the stone fragmentation associated with the ballistic or laser energy applied to cause stone migration. This can result in longer operative time, increased technical difficulty, potential loss of the stone in hydronephrotic kidney, and, ultimately, additional cost. ${ }^{12}$

Olbert et al. stated that there are three essential characteristics for an antiretropulsion device: it should avoid stone migration effectively, it should be easy to handle for the surgeon, and it should be resistant to damage by intracorporeal lithotripters. If damage to the system does happen, this should not alter the ability to be disengaged and removed smoothly. ${ }^{13}$

All of these devices yielded good outcomes in terms of better stone-free rate, but their additional cost actually limited significantly their dissemination worldwide. ${ }^{14,15}$ Where available, flexible URS remained the preferred way to check stone-free status at the end of the procedure.

When in proximity of the stone, lithotripsy is carried out. Regardless of the fact that ultrasonic and ballistic energy are still utilized in several developing countries, nowadays the gold standard for lithotripsy is represented by holmium laser. Laser setting is of uttermost important: as a matter of fact, by keeping the energy low and the pulse rate high, lithotripsy can be accomplished with limited retropulsion. The strategy of lithotripsy is also extremely important. Conversely to what is carried out in the kidney, in the ureter it is better to fire in the center of the stone creating a sort of cavity in it rather than firing peripherally so that the chance of thermal damage to ureteral mucosa is limited. Once fragments are generated, basketing is indicated to obtain the complete clearance of stones. Based on its properties and safety, tipless nitinol baskets are recommended for semirigid URS: baskets with fewer wires are indicated for removal of bigger fragments, whereas multiwires extractors are utilized when huge amount of tiny debris are the result of extensive lithotripsy. Of course, blind basketing should no longer be performed.

After completion of lithotripsy and litholapaxy, the integrity of the ureter is controlled endoscopically on the way out. This is an important step because as already widely demonstrated by randomized control trials (RCTs) and metaanalysis routine stenting is not indicated after uncomplicated URS. ${ }^{16}$ The use of steerable stents eases significantly their placement over a wire omitting final cystoscopy to do so. Also indwelling Foley catheter overnight can be omitted in case of uncomplicated URS without previous history of urinary infection.

\section{Postoperative care}

In cases where the procedure is uncomplicated, the patient could be discharged on the same day or at latest the day after. For uneventful procedures, short-term stenting is offered.

In case of fever, the antibiotic should be continued and the bladder catheter removed until the fever has broken to avoid vesicoureteral reflux and consequently to keep the pressure low into the collecting system.

\section{Outcomes}

In the Clinical Research Office of the Endourological Society (CROES) URS global study including 9681 patients, it was reported that semirigid URS was the most used procedure for ureteral stones in all locations.

The stone-free rates were $94.2 \%, 84.5 \%$, and $76.6 \%$ for distal, middle, and proximal ureteral stones, respectively.

For stones located in proximal ureter, failure and retreatment rates were significantly higher for semirigid URS compared with flexible URS.

The incidence of complications was low: $3.8 \%$ to $7.7 \%$ as intraoperative complications and $2.5 \%$ to $4.6 \%$ as postoperative complications.

Overall, this study did not show statistical difference in complication rate between semirigid and flexible URS. ${ }^{17}$

In general, semirigid URS provides an easier access to the lower ureter and facilitates multiple entries and exits during fragments retrieval. On the other hand, flexible URS represents the best way to get the access to the ureter in case of rigid and elevated bladder neck, huge prostates, narrow ureters and very developed psoas muscles. ${ }^{18}$

Consequently, the surgeon should choose the best ureteroscope for the stone and patient to be treated according also to the local availability, cost, personal preference, skill, and habit.

\section{Conclusions}

Semirigid URS is the gold standard of treatment for ureteral stones; standardization of the technique is the key to achieve optimal outcomes in a reproducible way. 


\section{Recommended Videos from Videourology}

1. Videourology 2011, Vol. 25, No. 4

Ureteroscopy Step by Step

Stephen Lukasewycz, Terri Deforge, Ricardo Miyaoka, Carl Sarkissian, Rakesh Khanna, and Manoj Monga

https://www.liebertpub.com/doi/10.1089/vid.2011.0001

2. Videourology 2015, Vol. 29, No. 5

Step by Step Flexible Ureteroscopy Supported with SPIES Technology for Conservative Treatment of UTUC

Guido Giusti and Silvia Proietti

https://www.liebertpub.com/doi/full/10.1089/vid.2015.0014

3. Videourology 2010, Vol. 24, No. 2

Digital Ureteroscopy: The Next Step

Sero Andonian, Zeph Okeke, and Arthur D. Smith

https://www.liebertpub.com/doi/full/10.1089/vid.2010.0025

\section{Author Disclosure Statement}

M.E.R.S., G.S., P.B., and F.G. have nothing to disclose; G.G. is consultant for Coloplast, Rocamed, Olympus, Lumenis, Boston Scientific, Cook Medical, and Quanta System; S.P. is consultant for Quanta System.

\section{Funding Information}

No funding was received for this article.

\section{Supplementary Data}

Supplementary Video S1 Full Supplementary Video Step 1 Supplementary Video Step 2 Supplementary Video Step 3 Supplementary Video Step 4 Supplementary Video Step 5 Supplementary Video Step 6 Supplementary Video Step 7 Supplementary Video Step 8 Supplementary Video Step 9

Supplementary Video Step 10

\section{References}

1. Türk, A. Neisius, A. Petrik, et al. Urolithiasis. EAU (European Association of Urology) Guidelines 2018.

2. Giusti G, Proietti S, Peschechera R. Sky is no limit for ureteroscopy: Extending the indications and special circumstances. World J Urol 2015;33:257-273.

3. Rane A, Sur R, Chew B. Retropulsion during intracorporeal lithotripsy: What's out there to help?. BJU Int 2010;106:591592.

4. Bourdoumis A, Stasinou T, Kachrilas S, et al. Thromboprophylaxis and bleeding diathesis in minimally invasive stone surgery. Nat Rev Urol 2014;11:51-58.

5. Kandasami SV, Mamoulakis C, El-Nahas AR, et al.; CROES URS Global Study Group. Impact of case volume on outcomes of ureteroscopy for ureteral stones: The clinical research office of the endourological society ureteroscopy global study. Eur Urol 2014;66:1046-1051.
6. Park HK, Paick SH, Oh SJ, Kim HH. Ureteroscopic lithotripsy under local anesthesia: Analysis of the effectiveness and patient tolerability. Eur Urol 2004;45:670-673.

7. Zeng G, Zhao Z, Yang F, et al. Retrograde intrarenal surgery with combined spinal-epidural vs general anesthesia: A prospective randomized controlled trial. J Endourol 2015;29:401-405.

8. Emiliani E, Talso M, Baghdadi M, et al. The use of apnea during ureteroscopy. Urology 2016;97:266-268.

9. Rukin NJ, Somani BK, Patterson J, et al. Tips and tricks of ureteroscopy: Consensus statement Part I. Basic ureteroscopy. Cent European J Urol 2015;68:439-446.

10. Türk C, Petřík A, Sarica K, et al. EAU guidelines on interventional treatment for urolithiasis. Eur Urol 2016;69:475-482.

11. Proietti S, Dragos L, Somani BK, et al. In vitro comparison of maximum pressure developed by irrigation systems in a kidney model. J Endourol 2017;31:522-527.

12. Elashry OM, Tawfik AM. Preventing stone retropulsion during intracorporeal lithotripsy. Nat Rev Urol 2012;9:691-698.

13. Olbert PJ, Keil C, Weber J, et al. Efficacy and safety of the Accordion stone-trapping device: In vitro results from an artificial ureterolithotripsy model. Urol Res 2010;38:41-46.

14. Sanguedolce F, Montanari E, Alvarez-Maestro M, et al.; EAU Young Academic Urologists- Endourology and Urolithiasis Working Group. Use of $\mathrm{XenX}^{\mathrm{TM}}$, the latest ureteric occlusion device with guide wire utility: Results from a prospective multicentric comparative study. World J Urol 2016;34:1583-1589.

15. Cabrera FJ, Preminger GM, Lipkin ME. Antiretropulsion devices. Curr Opin Urol 2014;24:173-178.

16. Haleblian G, Kijvikai K, de la Rosette J, et al. Ureteral stenting and urinary stone management: A systematic review. J Urol 2008;179:424-430.

17. Castro E, Osther P, Jinga V, et al. Differences in ureteroscopic stone treatment and outcomes for distal, mid-, proximal, or multiple ureteral locations: The clinical research office of the endourological society ureteroscopy global study. Eur Urol 2014;66:102-109.

18. Alameddine M, Azab M, Nassir A. Semi-rigid ureteroscopy: Proximal versus distal ureteral stones. Urol Ann 2016;8:84-86.

\section{Address correspondence to: \\ Guido Giusti, MD \\ Department of Urology \\ San Raffaele Hospital \\ Ville Turro Division \\ Via Stamira d'Ancona, 20 \\ Milan 20127 \\ Italy}

E-mail: drguidogiusti@gmail.com

$\begin{aligned} & \quad \text { Abbreviations Used } \\ & \text { CROES }=\begin{array}{c}\text { Clinical Research Office of the Endourological } \\ \text { Society }\end{array} \\ & \mathrm{CT}=\text { computed tomography } \\ & \mathrm{OR}=\text { operating room } \\ & \mathrm{PTFE}=\text { Polytetrafluoroethylene } \\ & \mathrm{RCTs}=\text { randomized control trials } \\ & \mathrm{UAS}=\text { ureteral access sheath } \\ & \mathrm{URS}=\text { ureteroscopy }\end{aligned}$

